\title{
A Postoperative Man with Marfan Syndrome with Palpitations and Chest Pain After Receiving the SARS-CoV-2 Vaccine
}

\author{
Kun $\mathrm{Li}^{1}{ }^{1} *$ \\ Bin Huang ${ }^{2, *}$ \\ Tao $\mathrm{Ji}^{3}$ \\ Shao-Gan $X u^{3}$ \\ Wen Jiang ${ }^{4}$
}

'Department of Anesthesiology, General hospital of central theater command of the People's Liberation Army, Wuhan, Hubei Province, 430070, People's Republic of China; ${ }^{2}$ The First School of Clinical Medicine, Southern Medical University, Guangzhou, Guangdong Province, 510515, People's Republic of China; ${ }^{3}$ Department of Thoracic Cardiovascular Surgery, General Hospital of Central Theater Command of People's Liberation Army, Wuhan, Hubei Province, 430070, People's Republic of China; ${ }^{4}$ Department of Health Medicine, General hospital of central theater command of the People's Liberation Army, Wuhan, Hubei Province, 430070, People's Republic of China

*These authors contributed equally to this work

\begin{abstract}
At the moment, the SARS-CoV-19 pandemic is still attacking the health of humanity, and vaccines are the primary health strategy to eradicate this global challenge. So, with the exception of the SARS-CoV-2 vaccine, no vaccine for any disease has been brought to clinical use so quickly. Therefore, even with strict management, it can still bring some special adverse effects. One of the most notable is the adverse cardiovascular reactions to SARS-CoV-2 vaccines. No case reports of individuals with irreversible arrhythmia complications following the SARS-CoV-2 vaccine have been found in the available literature. We report the first case of a postoperative man with Marfan syndrome with atrial fibrillation after receiving the SARS-CoV-2 vaccine.
\end{abstract}

Keywords: SARS-CoV-2, vaccine, adverse reactions, arrhythmology

\section{Introduction}

Corona virus disease 2019 (COVID-19) is spreading at an alarming rate from last year. The dominant infected patients had not yet been effectively controlled, and the invisible patients had come to light. ${ }^{1,2}$ Despite the confirmed COVID-19 patients have been strictly quarantined, protective measures have been taken for uninfected patients, such as masks, shelters and vaccination. ${ }^{2,3}$ So, hundreds of millions of Chinese have offered vaccination against SARS -CoV-2 with no fee. Government campaigns are needed to inform and persuade their citizens on the need for vaccination in the first half of 2021. ${ }^{4}$ At the same time, medical personnel have an obligation to disclose adverse reactions to SARS-CoV-2 vaccine. Vaccine hesitancy and vaccine desperately should be respected. Specific data should be provided for rare adverse reactions.

\section{Case Report}

A 31-year-old man presented to the cardiology clinic for palpitations and chest pain for approximately seven days. He had been diagnosed with Marfan syndrome at 24 years old and underwent the Bentall operation and mitral valve replacement. He felt almost no discomfort after this open-heart surgery. His three-kilometer running time was 15'35". He received an electrocardiogram to get his SARS-CoV-2 vaccination, and no arrhythmia was found (Figure 1A).

Then, he was given the SARS-CoV-2 vaccine (Vero Cell, Wuhan Institute of Biological Products Co., Ltd., $0.5 \mathrm{~mL}$ intramuscularly) with his doctor's
Correspondence: Wen Jiang; Shao-Gan Xu General hospital of central theater command of the People's Liberation Army, 627\#, Wuluo Road, Wuchangqu, Wuhan, Hubei, 430070, People's Republic of China Tel $+86 \quad 17720529297$

Fax +86 27-50772388

Email zbzqzyyjw@163.com;

whzyyxsg@163.com 

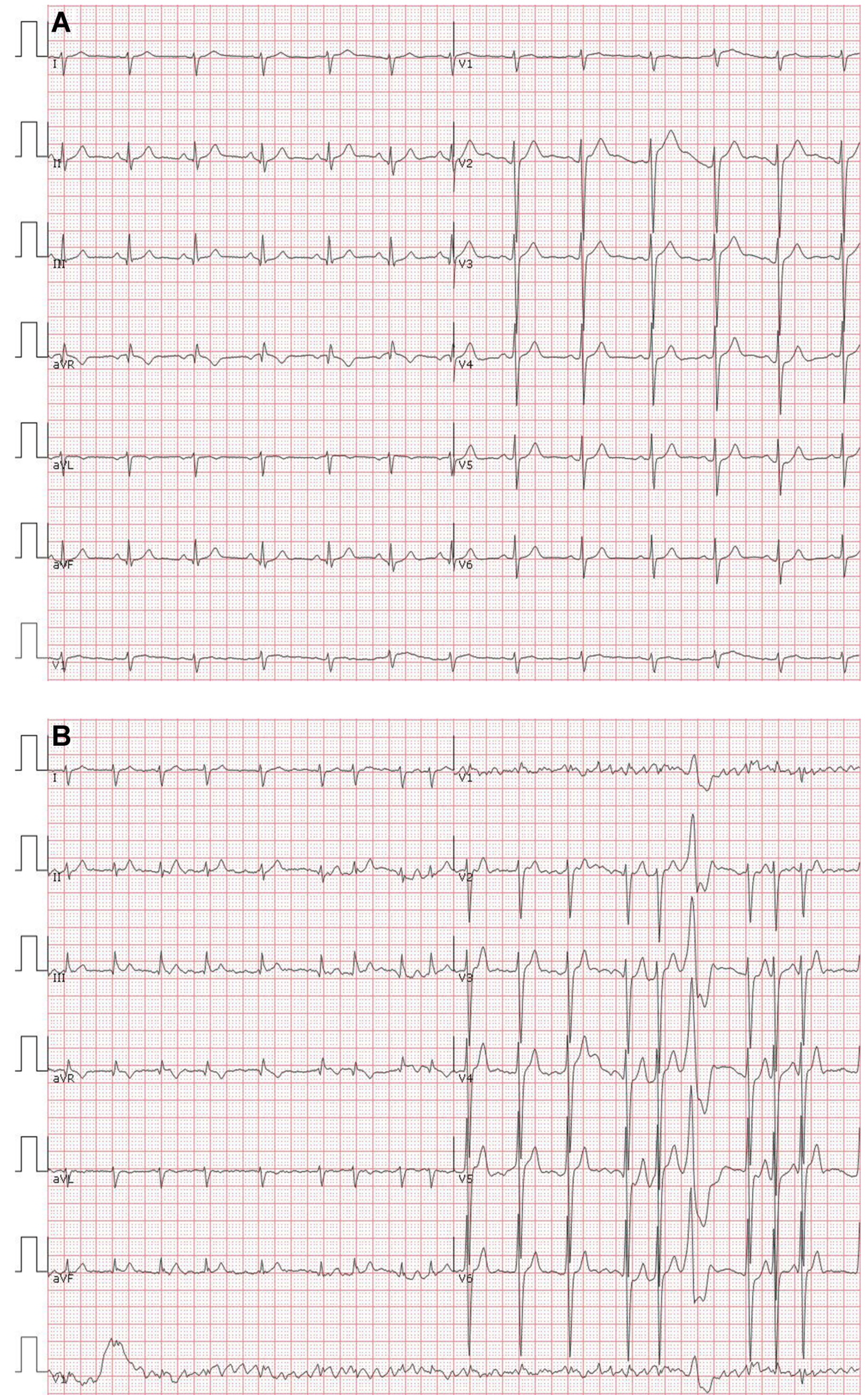

Figure I (A) Electrocardiogram findings before vaccination of SARS-CoV-2. (B) Electrocardiogram findings after vaccination of SARS-CoV-2. 
permission. Eight hours after he was vaccinated, he developed palpitations. Upon arrival at our clinic, chest pain continued. We performed another electrocardiogram, which showed atrial fibrillation with a rapid ventricular rate and occasional premature ventricular beats (Figure 1B).

In the following days, the patient received medication for sinus rhythm conversion and rate control. Unfortunately, the sinus rhythm of this patient was not restored.

\section{Discussion}

There are more than twenty vaccines in the clinical evaluation stage and at least 140 candidate vaccines in preclinical evaluation, including traditional inactivated or live attenuated virus vaccines, DNA or RNA vaccines, recombinant viral vector vaccines, and protein or peptide subunit vaccines. Safe and effective SARS-CoV-2 vaccines require further investigation. ${ }^{5,6}$ The independent board that conducted the interim analysis of Moderna's very large trial found that severe side effects included fatigue in $9.7 \%$ of participants, muscle pain in $8.9 \%$, joint pain in $5.2 \%$, and headache in $4.5 \%$. In the Pfizer/BioNTech vaccine trial, the numbers were lower: severe side effects included fatigue $(3.8 \%)$ and headache $(2 \%){ }^{7}$ No case reports of individuals with irreversible arrhythmia complications following the SARS-CoV-2 vaccine have been found in the available literature. However, 1226 reports of myocarditis after mRNA vaccination were received in the United States during December 29, 2020-June 11, 2021, from the Food and Drug Administration. ${ }^{8}$ This may be related to our case, but the mechanism is not clear.

Analysis of our case requires several considerations. This patient had Bentall operation and mitral valve replacement and possibly had an acute arrhythmia or upper respiratory tract infection. However, an upper respiratory tract infection cannot cause atrial fibrillation. When the vaccine is just injected, the first consideration should be the side effects of the vaccine.Our patient could not be explained by COVID-19 infection developing arrhythmias because there is no clinical history supporting a prior COVID-19 infection. ${ }^{9}$ In our case, we speculate that adverse reaction against the SARS-CoV-2 vaccine was responsible for the development of atrial fibrillation due to its temporal relationship. The mechanism of atrial fibrillation after receiving SARS-CoV-2 vaccine will require substantial evidences other than temporal aspects, such as careful measurement of biochemical and immunologic markers. Of course, it needs to be encouraged to report these adverse events by medical workers.

\section{Conclusion}

With the report of this case, we aim to emphasize that it is prudent to obtain the SARS-CoV-2 vaccine, especially in patients with heart disease who have undergone open-heart surgery. SARS-CoV-2 vaccine should be contraindicated in the patients whom were evident from ECG and not being able to restore the sinus rhythm in countries with low risk of infection, like China.

\section{Ethics Approval}

This research complies with the guidelines for human studies and is in accordance with the Declaration of Helsinki. The ethics Review Committee of General Hospital of Central Theater Command of the Chinese people's liberation army general hospital approved the use of clinical data of these patients in this study.

\section{Informed Consent}

Written informed consent was obtained from the patient for the publication. The patient provided written informed consent to participate in this study.

\section{Author Contributions}

All authors contributed to data analysis, drafting or revising the article, gave final approval of the version to be published, agreed to the submitted journal, and agreed to be accountable for all aspects of the work. These authors contributed equally to this work and should be considered as co-first authors: Kun Li and Bin Huang.

\section{Funding}

The authors declare that they have received no external funding for this study.

\section{Disclosure}

The authors report no conflicts of interest in this work.

\section{References}

1. Zhu J, Tan Y, Huang B, Zhu Y, Gao XH. Don't throw the stethoscope away! Eur Heart J. 2021;42:10-12.

2. Zhu J, Huang WC, Huang B, et al. Clinical characteristics and prognosis of COVID-19 patients with initial presentation of lung lesions confined to a single pulmonary lobe. Am J Transl Res. 2020;12:7501-7509.

3. Charitos IA, Del Prete R, Inchingolo F, et al. What we have learned for the future about COVID-19 and healthcare management of it? Acta Biomed. 2020;91:e2020126. 
4. Dal-Ré R, Stephens R, Sreeharan N. Let me choose my COVID-19 vaccine. Eur $J$ Intern Med. 2021;87:104-105. doi:10.1016/j. ejim.2021.01.030

5. Li L, Guo P, Zhang X, Yu Z, Zhang W, Sun H. SARS-CoV-2 vaccine candidates in rapid development. Hum Vaccin Immunother. 2021;17:644-653. doi:10.1080/21645515.2020.1804777

6. Charitos IA, Ballini A, Bottalico L, et al. Special features of SARS-CoV-2 in daily practice. World $J$ Clin Cases. 2020;8:3920-3933. doi:10.12998/wjcc.v8.i18.3920

7. Wadman M. Public needs to prep for vaccine side effects. Science. 2020;370:1022. doi:10.1126/science.370.6520.1022
8. Gargano JW, Wallace M, Hadler SC, et al. Use of mRNA COVID-19 vaccine after reports of myocarditis among vaccine recipients: update from the advisory committee on immunization practices - United States, June 2021. MMWR Morb Mortal Wkly Rep. 2021;70:977-982. doi:10.15585/mmwr.mm7027e2

9. Rajpal S, Tong MS, Borchers J, et al. Cardiovascular magnetic resonance findings in competitive athletes recovering from COVID-19 infection. JAMA Cardiol. 2021;6:116-118.

\section{Publish your work in this journal}

Infection and Drug Resistance is an international, peer-reviewed openaccess journal that focuses on the optimal treatment of infection (bacterial, fungal and viral) and the development and institution of preventive strategies to minimize the development and spread of resistance. The journal is specifically concerned with the epidemiology of antibiotic resistance and the mechanisms of resistance development and diffusion in both hospitals and the community. The manuscript management system is completely online and includes a very quick and fair peerreview system, which is all easy to use. Visit http://www.dovepress.com/ testimonials.php to read real quotes from published authors. 